\title{
Nivel de conocimiento de las medidas de racionalización del gasto farmacéutico: un estudio exploratorio
}

\section{M arta Pedraja I glesias*, Pilar RiveraTorres*, M ercedes M arzo N avarro**, Hernán Talledo Flores***}

\section{Introducción}

A lo largo de los últimos años, la sanidad ha sido considerada un objetivo de carácter social clave en la mayor parte de los países europeos, los cuales han pretendido ampliar sus prestaciones en cuanto a cantidad, cobertura y calidad. Aunque estos objetivos se han conseguido de forma notable, los procesos de descentralización sanitaria seguidos, el envejecimiento de la población así como las técnicas, cada vez más sofisticadas, necesarias para el tratamiento de ciertas enfermedades han elevado notablemente el gasto sanitario de estos páises.

Esta relevancia social de la atención de asistencia sanitaria pública y los elevados costes que deben asumir tradicionalmente las arcas del Estado por este concepto son dos realidades que han generado tanto una dotación presupuestaria anual al Sistema $N$ acional de Salud tendente al crecimiento como una tendencia al ahorro de consumos por vía legislativa. Entre las medidas le gislativas dirigidas al ahorro de consumos tomadas en los últimos años, destaca el Real D ecreto de Financiación Selectiva de M edicamentos de 1998, destinada al fomento del ahorro de financiación del consumo farmacéutico.

Este objetivo, la reducción del gasto farmacéutico, emerge como la razón principal de la existencia de Especialidades Farmacéuticas G enéricas o medicamentos genéricos (DiAZ, 2001).
D entro de este contexto, hay que hacer referencia a la Ley 13/1996, de M edidas Fiscales, Administrativas y de 0 rden Social, de 30 de diciembre de 1996 (modificación de la Ley 25/1990, de 20 de diciembre, del M edicamento), donde, en el art. 8 apartado 6 bis, se establece que la Especialidad Farmacéutica Genérica es «la especialidad con la misma forma farmacéutica e igual composición cualitativa y cuantitativa en sustancias me dicinales que otra especialidad de referencia, cuyo perfil de eficacia y seguridad esté suficientemente establecido por su continuado uso clínico. La especialidad farmacéutica genérica debe demostrar la equivalencia terapéutica con la especialidad de refe rencia mediante los correspondientes estudios de bioequivalencia», y al Real D ecreto 1035/1999, de 18 de junio, por el que se regula el sistema de Precios de Referencia en la financiación de medicamentos con cargo a fondos de la Seguridad Social o a fondos de estables afectos a la sanidad1. Este Real D ecreto define el precio de referencia como «la cuantía máxima que se financiará con cargo a fondos de la Seguridad Social 0 a fondos estatales afectos a la sanidad de las prestaciones de especialidades farmacéuticas incluidas en cada uno de los conjuntos homogéneos de terminadoss. En el caso de que el medicamento prescrito por el médico supere la cuantía establecida como precio de referencia, el farmacéutico deberá sustituirlo por una especialidad farmacéutica genérica del mismo conjunto homogéneo cuyo precio no supere al de referencia. En el caso de que el paciente desee el me dicamento recetado por el médico y no el genérico, deberá pagar la diferencia entre el precio de la especialidad farmacéutica dis- 
pensada y el de referencia fijado, además de efectuar, en su caso, la aportación económica que le pudiera corresponder.

Por lo tanto, para que se alcancen los ahorros estimados a través de la implantación de este sistema es necesario potenciar una cultura de genéricos entre los distintos actores implicados, médicos, farmacéuticos y pacientes, que ponga de manifiesto las ventajas del uso de las Especialidades Farmacéuticas G enéricas en relación a su coste y eficacia terapéutica (ROD Ríg UEZ y BigoRRA, 1999).

El correcto funcionamiento del sistema hace que sea necesario el cumplimiento de varias condiciones: i) que el gobierno establezca correctamente los grupos homogéneos y defina, sin lugar a dudas, qué es y cómo se calcula un precio de referencia; ii) que el médico confíe en los genéricos y se acostumbre a recetarlos tal como se describe en la Ley; iii) que el paciente confíe en el médico cuando éste le extienda una receta; y por último, iv) que el farmacéutico tenga flexibilidad para dispensar siguiendo las pautas del sistema, aś como para explicar, si fuese necesario, la causa de la sustitución de un medicamento por un genérico del mismo grupo homogéneo, o por el contrario el abono por parte del comprador de la diferencia de precios. Es de esperar que todas estas condiciones incidan en favorecer un mayor uso de los medicamentos genéricos dado que, hasta el momento, su utilización en el mercado farmacéutico español es muy baja?.

El trabajo que aquí se desarrolla tiene por objeto analizar las percepciones que posee uno de los principales agentes implicados, los ciudadanos, sobre el sistema de Especialidades Farmacéuticas $G$ enéricas y sobre los precios de referencia. Para ello se analiza la información proporcionada por el Centro de Investigaciones Sociológicas en 1999.

\section{M étodo}

\section{Base de datos}

La información analizada es la proporcionada por el Barómetro Sanitario, 1999 (3- O leada), realizado por el Centro de Investigaciones Sociológicas. Los datos fueron recogidos a través de la realización de una encuesta personal, de ámbito nacional, durante el mes de noviembre de 1999, siendo la ficha técnica del estudio la reflejada en el Cuadro 1.

La información recogida por el Barómetro Sanitario puede ser agrupada en dos grandes bloques: el relacionado con los medicamentos genéricos, y el relativo a los precios de referencia. La división del estudio en dos partes viene justificada porque, como

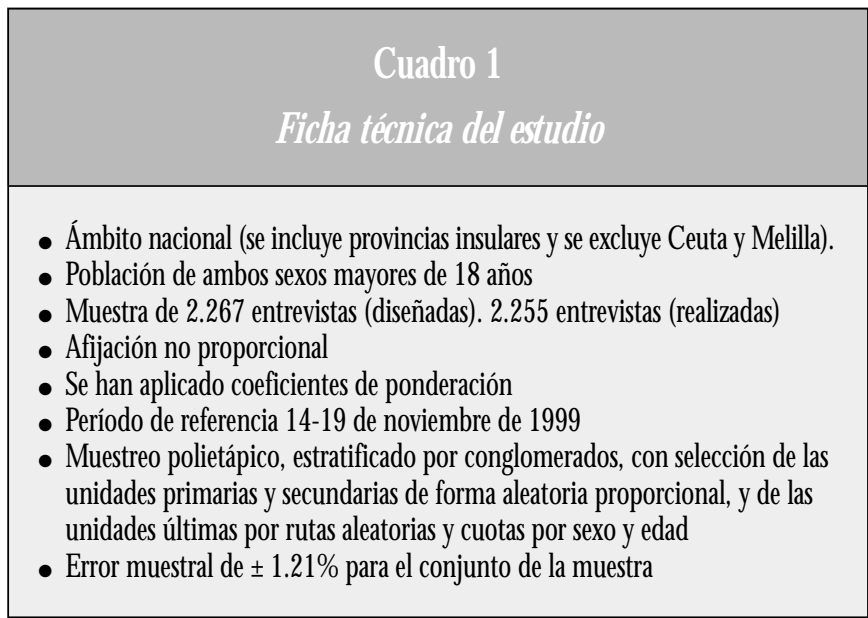

ya se ha puesto de manifiesto, los precios de referencia son sólo aplicados a los medicamentos genéricos, esto es, aquéllos que se comercializan bajo denominación genérica, una vez la marca de fantasía original pierde la patente sobre el principio activo (M EsTRE, 1999). Esto explica que, a pesar de ser dos términos estrechamente relacionados, se puedan analizar por separado.

Las escalas de medición utilizadas han sido distintas, en función del tipo de pregunta realizada, existiendo principalmente escalas dicotómicas y de interval 0 .

\section{Caracterización de la muestra}

El análisis de las características sociodemográficas de la muestra bajo estudio (Tabla 1) pone de manifiesto un equilibrio entre hombres y mujeres $(45,6 \%$ de hombres frente a $54,4 \%$ de mujeres). Atendiendo a la variable edad, el $59 \%$ de los individuos se sitúa en el intervalo de entre 18 y 49 años, mientras que casi un 20\% de la misma tiene más de 64 años. En lo relativo a su nivel de formación, tan sólo el 22,3\% han completado estudios secundarios o superiores, habiendo realizado la mayor parte de ellos únicamente estudios primarios $(63,2 \%)$. C on relación a su estado civil, predominan los casados $(60,7 \%)$, seguidos por los solteros $(29,1 \%)$. La mayor parte de los individuos analizados son usuarios activos del Sistema N acional de Salud $(77,4 \%)$, siendo el 22,6\% restante pensionistas.

\section{Resultados}

El nivel de conocimiento detectado sobre algunas de las potenciales medidas dirigidas a racionalizar el gasto en medicamentos en el presupuesto de la Sanidad Pública no puede considerarse alto (Gráfico 1), ya que a excepción de «Excluir determinados medicamentos de la prestación farmacéutica de la Seguridad Social», el resto de las medidas planteadas alcanza un nivel de co- 


\begin{tabular}{|c|c|c|c|c|}
\hline \multicolumn{5}{|c|}{$\begin{array}{l}\text { Tabla } 1 \\
\text { Características sociodemocráficas }\end{array}$} \\
\hline SEXO & \multicolumn{2}{|c|}{ M ujer } & \multicolumn{2}{|c|}{ Total } \\
\hline & \multicolumn{2}{|c|}{$\begin{array}{c}380 \\
54,4 \%\end{array}$} & \multicolumn{2}{|c|}{$\begin{array}{c}2.255 \\
100,0 \%\end{array}$} \\
\hline \multirow[t]{2}{*}{ EDAD } & $29 \geq X \geq 18$ & $49 \geq X \geq 30$ & $64<x$ & Total \\
\hline & $\begin{array}{c}555 \\
24,6 \%\end{array}$ & $\begin{array}{c}779 \\
34,5 \%\end{array}$ & $\begin{array}{c}441 \\
19,5 \%\end{array}$ & $\begin{array}{l}2.255 \\
100 \%\end{array}$ \\
\hline \multirow[t]{2}{*}{ NIVEL DE ESTUDIOS } & Sin Estudios & Primarios & Universitarios & Total \\
\hline & $\begin{array}{c}327 \\
14,3 \%\end{array}$ & $\begin{array}{l}1.423 \\
63,2 \%\end{array}$ & $\begin{array}{c}304 \\
13,5 \%\end{array}$ & $\begin{array}{l}2.255 \\
100 \%\end{array}$ \\
\hline \multirow[t]{2}{*}{ ESTADO CIVIL } & Soltero & Casado & Viudo & Total \\
\hline & $\begin{array}{c}656 \\
29,1 \%\end{array}$ & $\begin{array}{l}1.366 \\
60,7 \%\end{array}$ & $\begin{array}{c}171 \\
7,6 \%\end{array}$ & $\begin{array}{l}2.255 \\
100 \%\end{array}$ \\
\hline \multirow[t]{2}{*}{ TIPO DE USUARIO } & \multicolumn{2}{|l|}{ Activo } & \multicolumn{2}{|l|}{ Total } \\
\hline & \multicolumn{2}{|l|}{$\begin{array}{l}1.742 \\
77,4 \%\end{array}$} & \multicolumn{2}{|c|}{$\begin{array}{l}2.255 \\
100 \%\end{array}$} \\
\hline
\end{tabular}

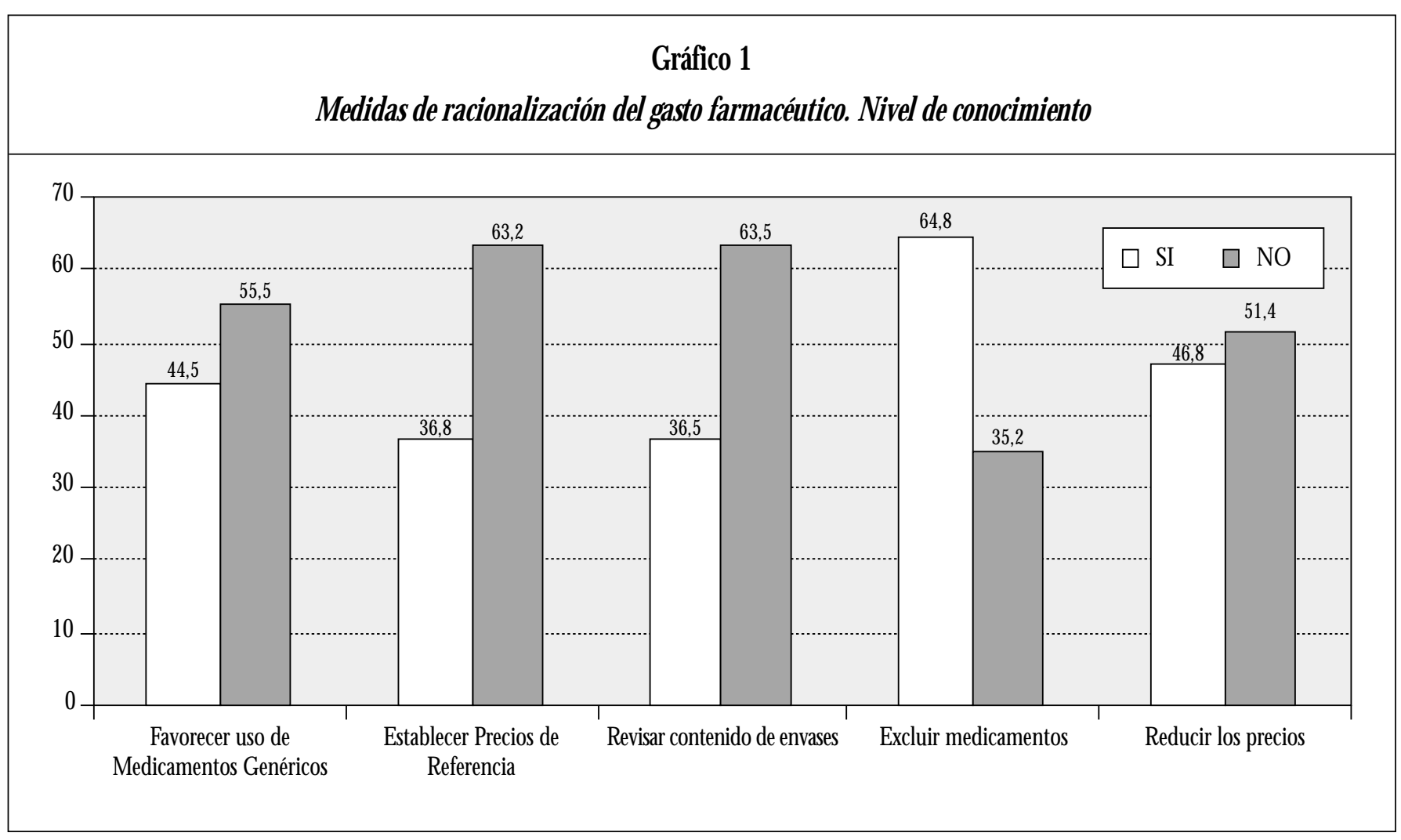

Fuente: Elaboración propia. 


\section{Gráfico 2}

Fuente de información empleada sobre las medidas de racionalización del gasto en medicamentos

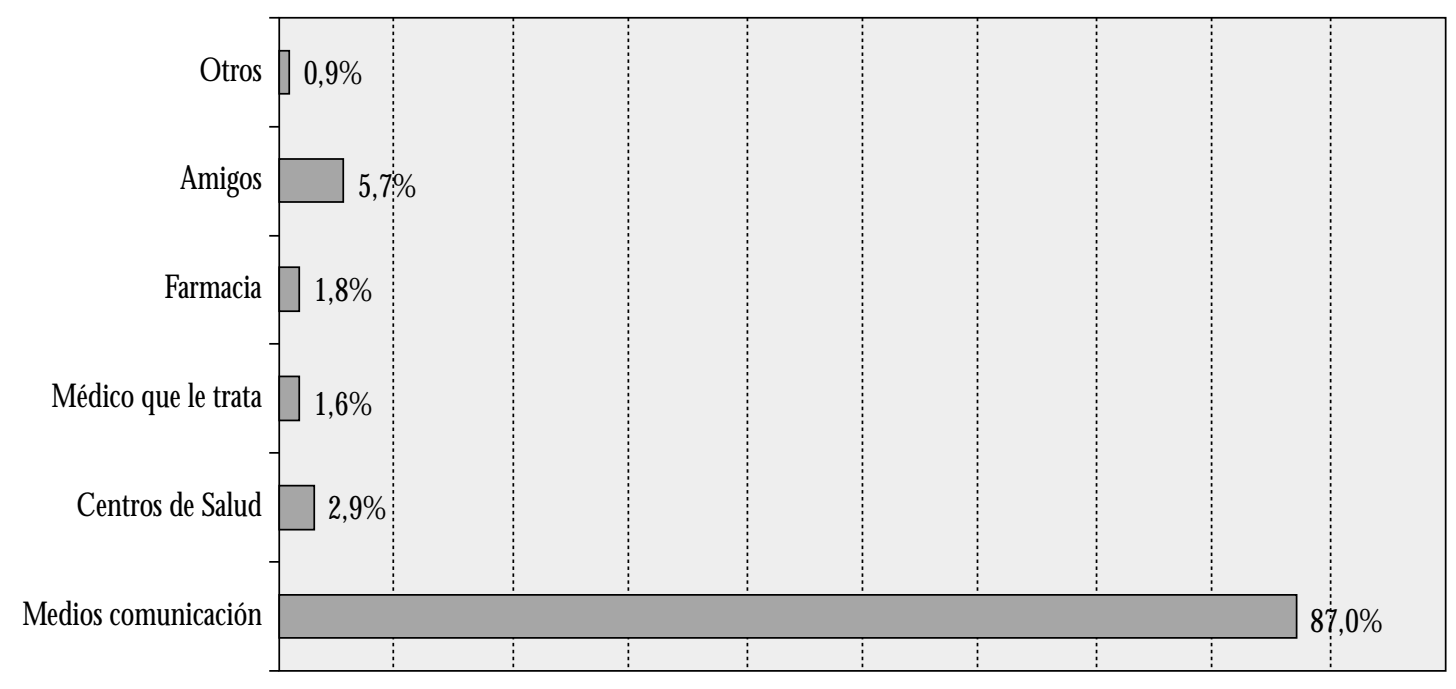

Fuente: Elaboración propia.

nocimiento en torno al $35 \%$. Cabe resaltar que la medida relacionada con el establecimiento de precios de referencia, tema objeto de estudio en el presente trabajo, presenta el segundo nivel más bajo de conocimiento, ya que tan sólo el $36,8 \%$ de la muestra afirman conocer dicha medida.

Los individuos que manifiestan conocer alguna de las medidas planteadas anteriormente, pueden ser caracterizados 3 a través de sus características sociodemográficas. Así, estos individuos tienen entre 30 y 49 años, estudios secundarios 0 universitarios, un nivel de renta mensual por unidad familiar situado entre 1202.02 y 1803.04 euros, y son usuarios activos del Sistema $\mathrm{Na}$ cional de Salud. Por su parte, aquellos que manifiestan no haber oído hablar de ninguna de las medidas, se caracterizan por lo contrario: más de 50 años, bajo nivel tanto de estudios como de renta, y no son usuarios activos del Sistema N acional de Salud.

La información sobre las anteriores medidas ( $G$ ráfico 2 ) ha sido obtenida fundamentalmente a través de los medios de comunicación (prensa, radio y televisión). Cabe destacar la baja incidencia del papel desempeñado como fuente de información por médicos y farmacéuticos, ya que en ninguno de los casos lle ga al $2 \%$.

Para poder detectar la percepción de la eficacia de una de las medidas planteadas, «Favorecer el uso de medicamentos genéricoss, y dado el bajo conocimiento esperado de este concepto, se proporcionó a los individuos analizados una tarieta con su definición4. Tras leer dicha definición, el 67,7 \% de la muestra ma- nifestó que esta medida les parecía bastante o muy eficaz para conseguir el objetivo de racionalizar el gasto en medicamentos.

Cabe destacar que un 19\% de los encuestados optó por la respuesta «N o lo sé». Esta respuesta fue proporcionada principalmente por mujeres de más de 64 años, viudas, con bajos niveles de ingresos, - inferiores a 601,01 euros al mes- , sin estudios y que no son usuarias activas del Sistema Nacional de Salud. Por el contrario, aquellos que la consideraron como una medida muy eficaz, fueron principalmente los solteros, entre 30 y 49 años, con niveles de ingresos de la unidad familiar superiores a 1.202,02 euros al mes, con estudios superiores no universitarios o universitarios y usuarios activos del Sistema N acional de Salud.

Además, la cantidad de medicamentos genéricos ofertados en el mercado español es desconocida por la mayor parte de los encuestados $(59,3 \%)$. Este nivel de desconocimiento es más patente en las mujeres, viudas y casadas, con niveles de ingresos inferiores a 600,01 euros al mes, que carecen de estudios, mayores de 50 años, y que en la actualidad son pensionistas.

Este bajo nivel de conocimiento sobre la existencia de gené ricos en el mercado español, se relaciona con el bajo nivel de compra manifestado por la muestra. Aśi, únicamente el 16,5\% manifiesta que ha comprado o tomado genéricos en alguna ocasión, destacando el gran porcentaje de personas que no lo han hecho (52,9\%). Los individuos que manifiestan haberlos tomado son personas entre 18 y 49 años, solteras, con estudios secun- 


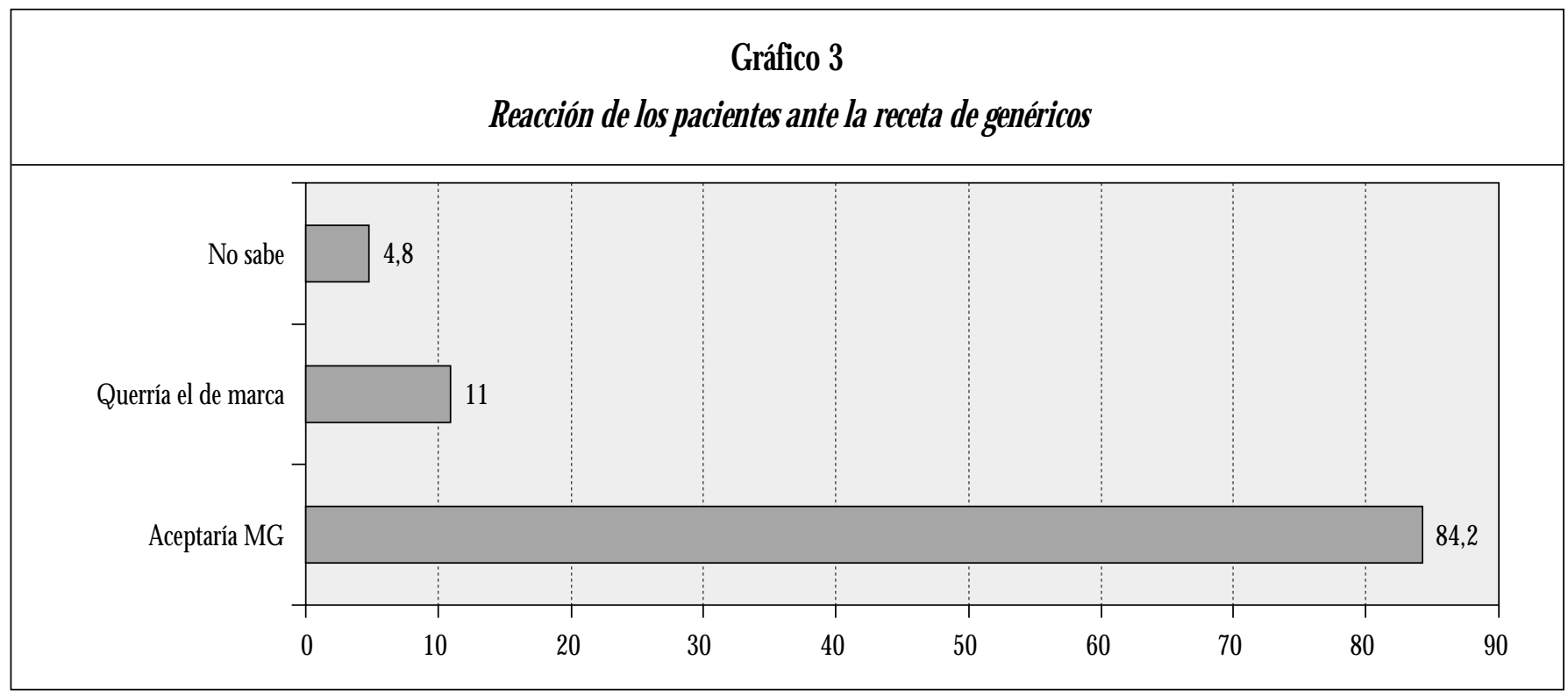

Fuente: Elaboración propia.

darios o estudios universitarios, usuarios activos del Sistema Nacional de Salud y con un alto nivel de ingresos.

Para poder detectar la opinión de los individuos analizados sobre el papel como fuente de información que podrían desempeñar los farmacéuticos, se les preguntó sobre la obligación del farmacéutico de informar de la existencia de un medicamento genérico que tenga las mismas características que otro de marca, pero con un precio más reducido. El 81,9\% manifestó que los farmacéuticos deberían informar de esta situación. Este conjunto de individuos eran solteros, jóvenes y de mediana edad, con ingresos entre 1.202,02 y 1.803,04 euros al mes y usuarios activos del Sistema N acional de Salud.

Con el objetivo de determinar cuál sería la reacción de los individuos analizados a los medicamentos genéricos, se les ponía en la situación de que, tras visitar al medico, éste les recetara un medicamento genérico, explicándoles que se trata del mismo medicamento en cuanto a su eficacia, forma de administración y composición, en lugar de uno de marca que el paciente ya conocía. El $84,2 \%$ de los individuos manifestaron que aceptarían de buen grado el genérico, y sólo un 11\% trataría de conseguir que se le recetase el de marca (Gráfico 3).

Los individuos que manifestaron aceptar el genérico pueden ser caracterizados como individuos jóvenes, con altos niveles de renta y de estudios, solteros y usuarios activos del Sistema $\mathrm{Na}$ cional de Salud. Por su parte, aquellos que indicaron preferir el medicamento de marca se caracterizan por ser separados o viudos, con ingresos mensuales de la unidad familiar inferiores a 601.01 euros, con más de 64 años, sin estudios y jubilados.
A aquellos individuos que manifestaron aceptar la receta del genérico, se les preguntó sobre dos de las posibles causas que les llevaría a aceptarlo: i) se lo recetó el médico en el cual confía; y, ii) porque siendo igual de eficaz, es más barato. Las respuestas dadas ponen de manifiesto el importante papel que puede desempeñar el médico para que el sistema de genéricos funcione correctamente, ya que el $61,4 \%$ de los encuestados seleccionó la primera causa. Estos individuos que ponen de manifiesto su confianza en el médico son, principalmente, los viudos y casados de mayor edad, sin estudios, con un bajo nivel de ingresos, y que actualmente están jubilados. Por su parte, aquellos cuya principal razón para aceptar el genérico era su precio son los individuos solteros, jóvenes, con estudios universitarios, altos niveles de ingresos, y usuarios activos del Sistema N acional de Salud.

Por su parte, a aquellos individuos que manifestaron no aceptar el genérico y que tratarían de conseguir que se les recetase el de marca, se les preguntó también por dos de sus posibles razones: i) lo he tomado en otras ocasiones y me ha ido bien; y ii) no confío en los medicamentos más baratos. La razón mayoritaria resultó ser la primera opción (66,3\%).

En relación al análisis del nivel de conocimiento y opiniones sobre los precios de referencia, el esquema seguido es similar al anterior. Así, lo primero que se analizaba era el nivel de conocimiento de los individuos sobre la existencia del Real Decreto 1035/1999, de 18 de junio, por el que se regula el sistema de Precios de Referencia en la financiación de medicamentos con cargo a fondos de la Seguridad Social 0 a fondos de estables afectos a la sanidad, resultando que únicamente el $26 \%$ tenía conocimiento del mismo. El nivel de conocimiento era superior en 


\section{Gráfico 4}

O pinión sobre la medida de los precios de referencia

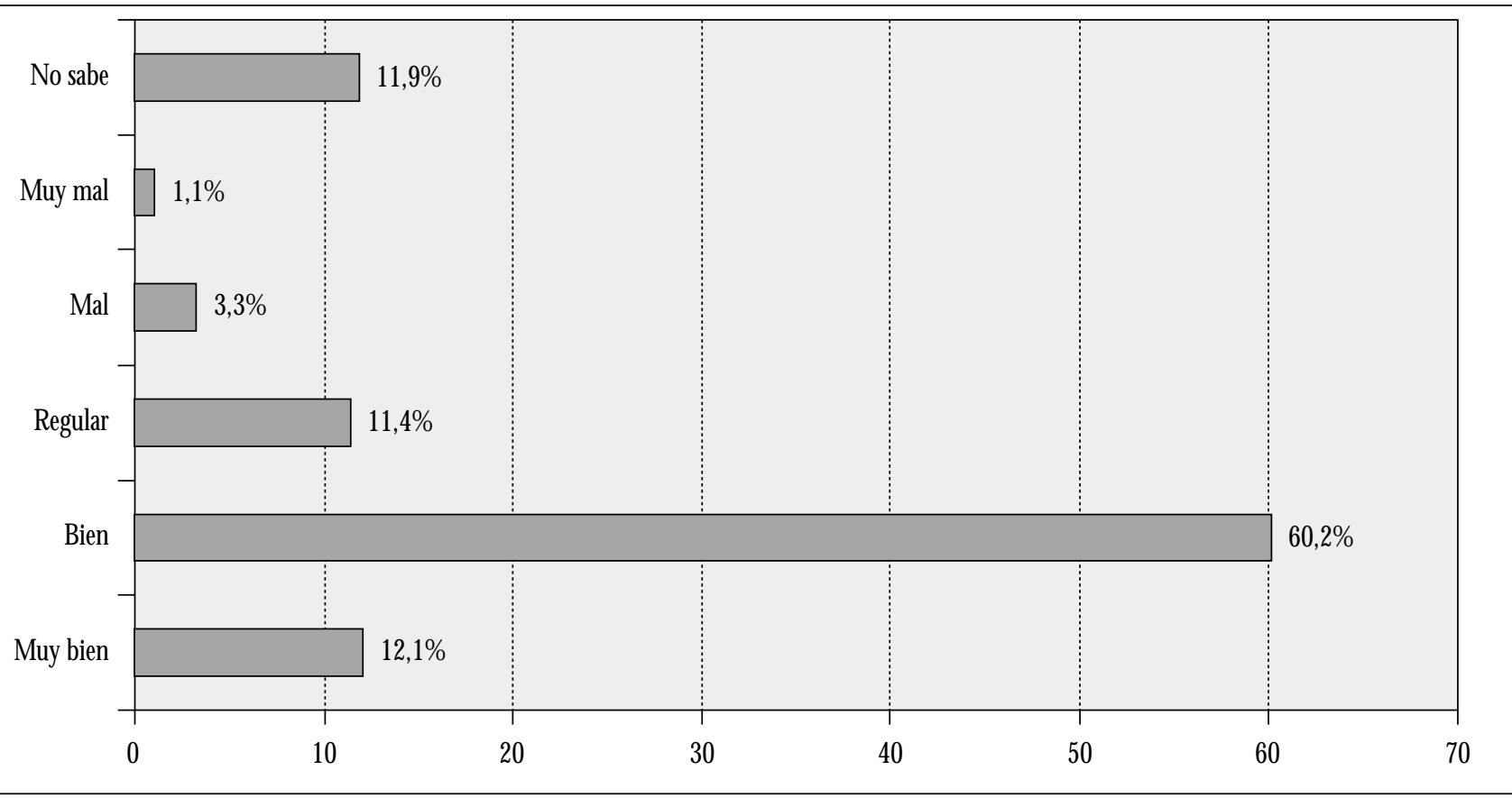

Fuente: Elaboración propia.

los hombres, entre los 30 y 49 años, usuarios del Sistema N acional de Salud, con altos niveles educativos y de ingresos. Por contra, aquellos que no lo conocían, eran principalmente las muje res de mayor edad, menor nivel educativo así como de ingresos y jubiladas.

Con independencia de que el entrevistado conociera o no la existencia del Real D ecreto descrito anteriormente, se les leía una tarjeta donde se les explicaba en qué consistía dicho Decreto5. Una vez proporcionada esta información, se les preguntó su opinión sobre la medida de establecer precios de referencia, tal y como estaba implantado en otros países europeos. En general esta medida era percibida como favorable por la mayor parte de la muestra, ya que el $72,3 \%$ manifestaron que les parecía bien 0 muy bien (Gráfico 4). Estos individuos pueden ser caracterizados como personas de entre 30 y 49 años, separados, con altos niveles de estudios y de renta, y usuarios activos del Sistema $\mathrm{Na}$ cional de Salud.

Sin embargo, este nivel de acuerdo baja a la hora de analizar la eficacia de la medida propuesta para reducir el gasto público en medicamentos. Así, la mitad de la muestra indicó que dicha medida era bastante o muy eficaz, mientras que casi una cuarta parte de la misma manifestó no saberlo (Gráfico 5). Esta falta de opinión es más patente en las mujeres de más de 64 años, viudas, que carecen de estudios, con bajos niveles de ingresos y que no son usuarias activas. Por el contrario, los que piensan que esta medida logrará muchos o bastante ahorros se caracterizan por ser mas jóvenes, con altos niveles de estudios y de ingresos.

Las percepciones existentes sobre el hecho de que los farmacéuticos pudieran sustituir medicamentos previamente recetados por un médico por un medicamento genérico con su precio de referencia, son bastante favorables, ya que esta medida les parecía bien o muy bien a casi al $70 \%$ de la muestra, siendo los individuos jóvenes, con mayores niveles de estudios y de ingresos los que más apoyaban esta medida.

Para detectar los posibles comportamientos de la muestra ante la existencia de los precios de referencia, se le ponía a los individuos analizados en la situación de que, tras una visita al mé dico, éste le recetase una medicina más cara que la que paga la Seguridad Social a través del precio de referencia. Las opciones planteadas fueron, principalmente, tres: i) aceptar que el farmacéutico la sustituyera por la de precio de referencia; ii) pagar la diferencia; $y$, iii) volver al médico para que le recetase otra dentro del precio de referencia. Tal y como se observa en el G ráfico 6 , se corrobora el resultado obtenido anteriormente sobre el papel a desempeñar por el farmacéutico, ya que la mitad de la muestra estaría dispuesta a aceptar el cambio de medicamento. Por su parte, el 17\% manifestó que pagaría la diferencia mientras que casi una cuarta parte de la muestra volvería al médico. 


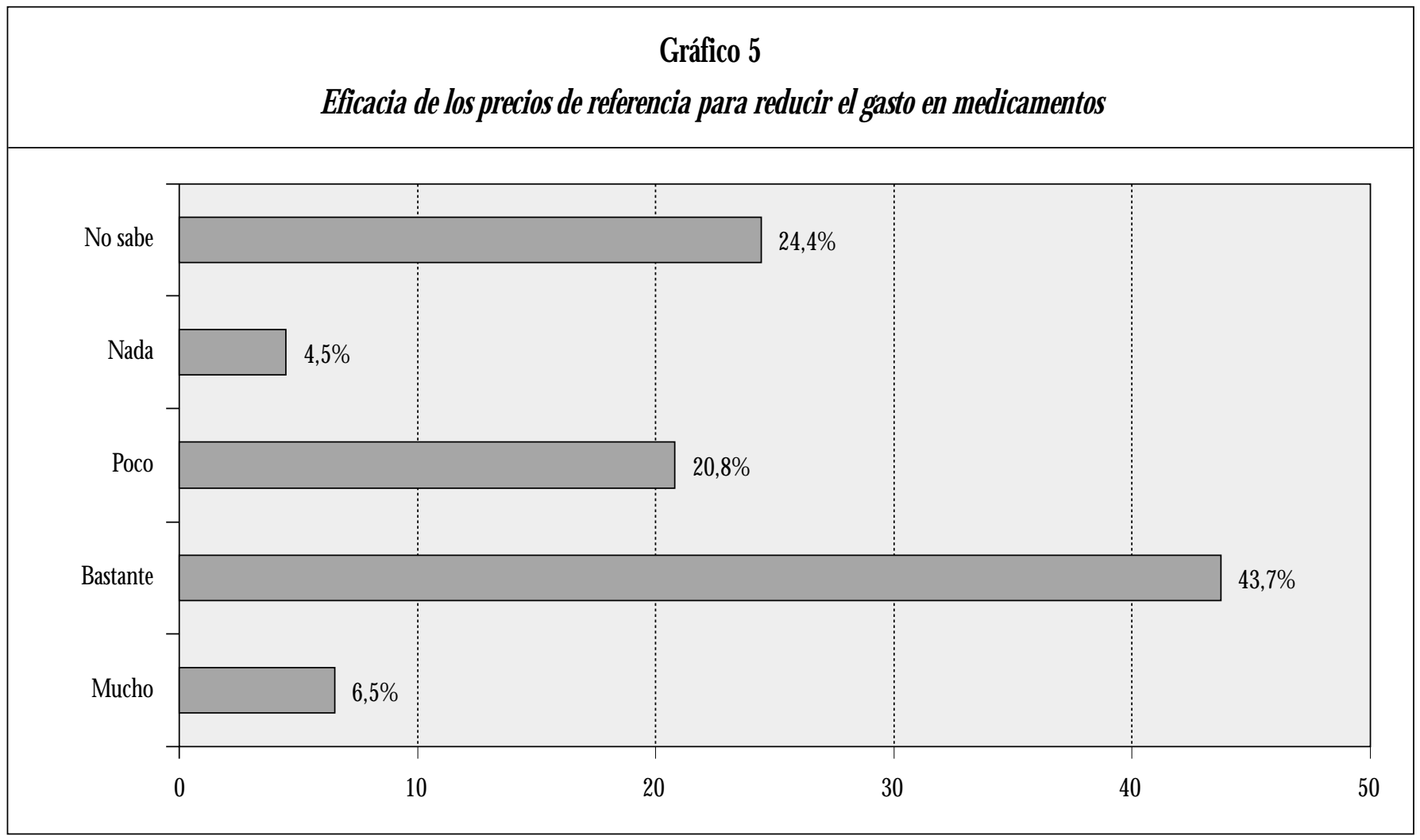

Fuente: Elaboración propia.

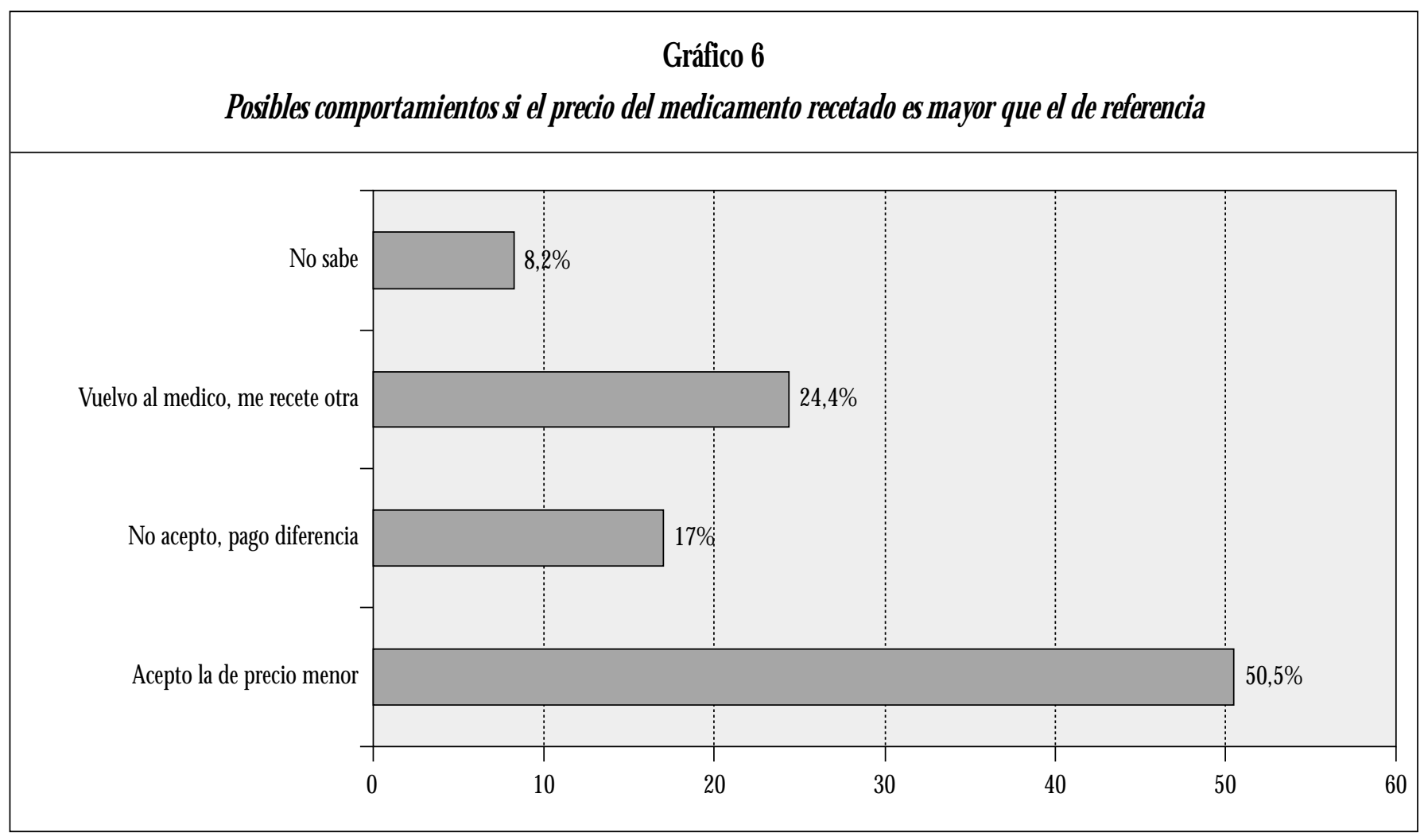

Fuente: Elaboración propia. 
I gual que en el caso anterior, los individuos que aceptarían el cambio de medicamento son jóvenes, con al tos niveles de estudios y de renta, mientras que los individuos que volverían a visitar al médico son la personas de mayor edad, con menor nivel de ingresos, y jubilados.

A modo de resumen se podría decir que, en general, el conocimiento de la existencia de medicamentos genéricos y de los precios de referencia es bajo, siendo inferior en las personas de mayor edad, con menor nivel de estudios, y de renta, que no son usuarias activas del Sistema N acional de Salud. Además, la percepción de los beneficios del sistema planteado, así como el conocimiento de su funcionamiento es mayor en las personas jóvenes, con mayor nivel de renta y de estudios, que en la actualidad son usuarios activos del Sistema N acional de Salud.

\section{Conclusiones}

Los resultados obtenidos a través de la realización de este trabajo pretenden ser una primera aproximación a las percepciones sobre los medicamentos genéricos y los precios de referencia manifestados por los pacientes potenciales, en los inicios de implantación del sistema.

En general, podemos establecer la existencia de un bajo nivel de conocimiento sobre las posibles medidas dirigidas a racionalizar el gasto en medicamentos en el presupuesto de la Sanidad Pública, especialmente sobre las dos medidas aquí analizadas, fa- vorecer el uso de medicamentos genéricos y establecer un precio de referencia para al gunos medicamentos. Este desconocimiento queda también patente al determinar la eficacia percibida de estos conceptos para reducir el gasto en medicamentos.

El resultado más destacable del estudio pone de manifiesto la existencia de un grupo de individuos, formado principalmente por la gente de mayor edad, con bajos niveles de ingresos y estudios, que no son usuarios activos del Sistema N acional de Salud. Estas características se corresponden con el perfil de los pensionistas, quienes manifiestan tener un menor conocimiento de los conceptos analizados y unas percepciones inferiores sobre los beneficios derivados de los mismos. Este resultado se ve corroborado por otros estudios previos relacionados con riesgo percibido asociado a la compra de genéricos. Así, en función de la variable edad, se ha detectado la existencia de una relación positiva entre ésta y el riesgo (Wu et al., 1984; M ITCHELL y BoustANI, 1993; Díaz et al., 2001), de tal manera que «a mayor edad, mayor riesgo percibido en el consumo de medicamentos sin marca».

Dado el elevado porcentaje de población que representa el segmento de jubilados en las sociedades modernas, se hace necesario implantar campañas de comunicación más eficaces, por parte de la Administración Pública, dirigidas a este grupo de ciudadanos. Estas campañas informativas deben centrarse en dar a conocer la existencia de los medicamentos genéricos y de los precios de referencia, así como explicar, de la forma más sencilla posible, su funcionamiento. Además de los medios de comunicación masivos, se ría necesario fomentar el papel de la comunicación personal desempeñado por el médico dada la confianza que este grupo de individuos deposita en esta figura.
* Facultad de Ciencias Económicas y Empresariales de la Universidad de Zaragoza. ** Facultad de H umanidades y Ciencias Sociales de la U niversidad de Zaragoza.

*** Facultad de Ciencias Económicas y Empresariales. Universidad Complutense de Madrid.

1 La aprobación del precio del genérico obliga a que éste tenga un precio entre un 25-30\% menor al del precio del producto original, basándose en que este tipo de productos no tiene costes ligados a la investigación; ya que se comercializan cuando ha finalizado el periodo de protección de la patente de los medicamentos innovadores (VILLALUENGa, 2001).

2 D esde noviembre de 1997, momento de la aparición de la primera Especialidad Farmacéutica Gerérica en España, dicha cifra no supera el 2\% (ALmIRALL, 1998; EuROPEAN GENERIC MEDICINES AsSOCIATION, 1999).

$3 \mathrm{H}$ an sido realizadas las tablas de contingencia con sus correspondientes residuos ajustados, así como el valor estadístico Chi-cuadrado para comprobar la significatividad de las diferencias existentes.

4 La definición proporcionada fue la siguiente: «un medicamento genérico es aquel cuya composición y presentación es idéntica al original de determinada marca, con la misma eficacia y calidad, pero más barato ya que transcurridos 10 años, tiempo durante el cual el laboratorio investigador tiene la exclusiva para comercializar un producto y amortizar los elevados gastos de investigación, la Ley permite que otro laboratorio, previa solicitud al M inisterio de Sanidad y Consumo, pase a comercializarse con el nombre del componente activo del producto, seguido del nombre del laboratorio y un distintivo, lo que lleva consigo una rebaja en el coste del producto, al no tener gastos de investigación».

5 «.. el Real D ecreto sobre Precios de Referencia consiste en establecer un precio máximo por cada grupo homogéneo de medicamentos, es decir, con la misma composición cuantitativa, cualitativa, dosis, vía de administración y presentación. De tal forma que si el medicamento prescrito por el médico es igual o demenor precio queel de referencia, el paciente obtendrá el fármaco recetado. Si, por el contrario, el medicamento prescrito es más caro que el de referencia, el farmacéutico informará de la existencia de otro medicamento más barato y el paciente podrá optar por sustituirlo por éste de menor precio o por abonar la diferencia de precio entre el recetado y el de referencia». 


\section{Bibliografía}

AlmiRall, M . (1998): «Aplicación de una política de genéricos y su impacto en la cadena sanitaria», La Revista Española de Farmacología, Año 4, no 1.

Díaz, A., González, C., González, S., Suárez, A. y Trespalacios, J. A. (2001): «Un estudio exploratorio de la intención de compra de medicamentos genéricos», Actas del XI Congreso N acional de ACEDE, Zaragoza, septiembre, 2001. CD.

DiAz, J. (2001): «M edicamentos genéricos y oficina de farmacia; en SALGAD O, A.: M edicamentos genéricos: realidad y perspectivas, N ovartis, Laboratorios Géminis. www.webgenéricos.com

European Generic Medicines Association (1999): Facts and Figures, Healthcare $M$ arkets, www. egagenerics.com.

M ESTRE, J., (1999): «La relación entre un sistema de Precios de referencia y M edicamentos genéricos», H acienda Pública Española, 150, pp. 173-179.
M ITCHELL, V. y BoustANI, P. (1993): «T he effect of demographic variables on measuring percieved risk» en LEVY, M . y GREWAL. D. (eds.), Academy of M arketing Science Conference, D evelopment in M arketing Science, vol. 16, mayo, pp. 663-669.

Rodríguez, M . y Bigorra, J. (1999): «Productos G enéricos en España», en M edicamentos Genéricos. Realidad y Perspectivas. www.webgenéricos.com

Villaluen GA, J., (2001): «Herramientas de M arketing en la Industria Farmacéutica en España», Investigación y M arketing, no 73 diciembre, pp. 4.954.

Wu, B., H OLM ES, J. y ALEXANDER, J. (1984): «Risk taking: its effects on selection of branded and generics grocery items», Akron Business and Economic Review, vol. 15, n-3, pp. 12-17. 
